\title{
Magnetic Fields in Strongly Interacting Galaxy Systems
}

\author{
Krzysztof T. Chyży \\ Astronomical Observatory, Jagiellonian University, Kraków, Poland \\ Rainer Beck \\ Max-Planck-Institut für Radioastronomie, Bonn, Germany
}

\begin{abstract}
We obtained VLA radio total power and polarization maps of a merging pair of galaxies NGC4038/39. The whole system is filled with bright extended radio emission with a high thermal fraction of $\simeq 50 \%$ at $10.45 \mathrm{GHz}$ and very strong magnetic fields of $\simeq 20 \mu \mathrm{G}$. A star-forming region at the southern end of the dark cloud complex extending between the galaxies has highly tangled magnetic fields reaching the strength of $\simeq 30 \mu \mathrm{G}$, even larger than in both individual galaxies. The polarized components associated with the HI ridge at the base of one of the tidal tails and with the northern complex of dark clouds (weakly forming stars) have been identified. A relic magnetic spiral coincident with the northern galaxy has been found, too. Our data on the magnetic fields combined with those in $\mathrm{HI}, \mathrm{H} \alpha$, X-rays and in far infrared are used as a diagnostic tool to distinguish several physically distinct regions.
\end{abstract}

\section{When galaxies collide}

We present the first study of magnetic field structure in galaxies in a merging state. We obtained for NGC 4038/39 (the Antennae) VLA radio total power and polarization maps at $8.44 \mathrm{GHz}, 4.86 \mathrm{GHz}$ and $1.49 \mathrm{GHz}$. The strong radio emission (Fig. 1) is found to be associated with both galaxy disks and with the southern part of the "overlapping region" extending between galaxies where vigorous star-formation is present. The thermal fraction of NGC 4038/39 is found to be about $50 \%$ at $10.45 \mathrm{GHz}$, higher than in normal spirals.

In the NE disk a radio total power ridge extends far out along the southern tidal tail. The ridge is polarized and the polarization degree increases along this feature from about $5 \%$ in its northern part to about $40 \%$ at its southernmost tip. Magnetic fields which are aligned with the tidal tail form a rudimentary spiral in the NW part. The B-vectors (not corrected for Faraday rotation which is $\leq 15^{\circ}$ ) are inclined there by large angle of about $45^{\circ}$ with respect to the total power feature, which is not observed in normal spirals. Close to the centre of the northern galaxy the B-vectors are almost radial, following local dust lanes. Clear traces of another spiral with a very large pitch angle are present around the nucleus of the southern galaxy. 


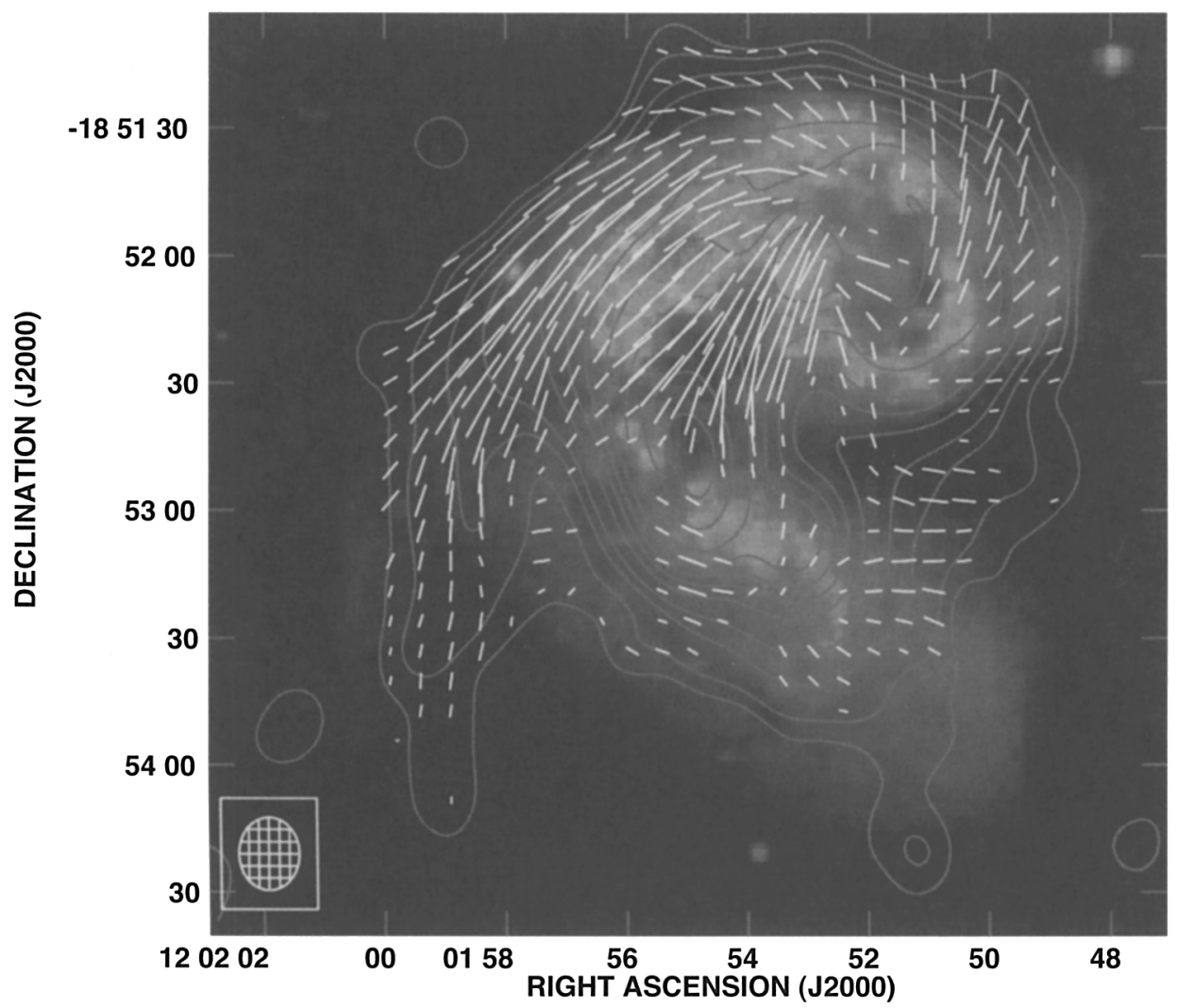

Figure 1. Contours of total power emission and apparent B-vectors of polarized intensity of NGC $4038 / 39$ at $4.86 \mathrm{GHz}$ made from a combination of VLA data in C and D arrays overlaid upon the DSS image

\section{Recycling magnetic fields}

Our calculations show (Chyży et al. 2002, Chyży \& Beck 2003) that the total magnetic fields of the individual Antennae galaxies and of the "overlapping region" are about twice stronger than in normal spirals. Locally, the strongest magnetic fields of $B_{\text {tot }} \simeq 30 \mu \mathbf{G}$ are observed outside the galaxy disks, in the "overlapping region" between them, a phenomenon which was never observed before in any galaxy system. This strong magnetic fields are probably the result of vivid star-formation and enhanced gas turbulence and/or compression of original fields pulled out from the parent disks.

Regular magnetic fields in NGC 4038/39 are similar to that observed in normal spirals and are of order of $3 \mu \mathrm{G}$. However, the field regularity is extremely low (0.1-0.2), in contrast to the typical value of 0.5 found for normal galaxies (Beck et al. 1996). This means that in the interacting system either strong amplification of turbulent fields occurs, or both turbulent and regular 
magnetic fields are generated but the regular fields are much more tangled in the starburst regions than in normal spirals.

With the help of the radio total power and polarization data we are also able to identify several large domains in the system with different physical conditions and star-forming regions in different evolutionary stages.

Acknowledgments. This work was supported by a grant from the Polish Research Committee (KBN), grant no. PB249/P03/2001/21

\section{References}

Beck R., Brandenburg A., Moss D. et al. 1996, An.Rev.A\&A, 34, 155

Chyży K.T., Soida M., Urbanik M. \& Beck R. 2002, AP\&SS, 281, 409

Chyży K.T., Beck T. 2003, AA submitted 